Article

\title{
A Lipid-Coated Nanoconstruct Composed of Gold Nanoparticles Noncovalently Coated with Small Interfering RNA: Preparation, Purification and Characterization
}

\author{
Anna V. Epanchintseva ${ }^{\dagger}$, Julia E. Poletaeva ${ }^{\dagger}$, Ilya S. Dovydenko ${ }^{+}\left(\mathbb{D}\right.$, Boris P. Chelobanov, Dmitrii V. Pyshnyi $\mathbb{D}^{\text {, }}$ \\ Elena I. Ryabchikova *(i) and Inna A. Pyshnaya *(i)
}

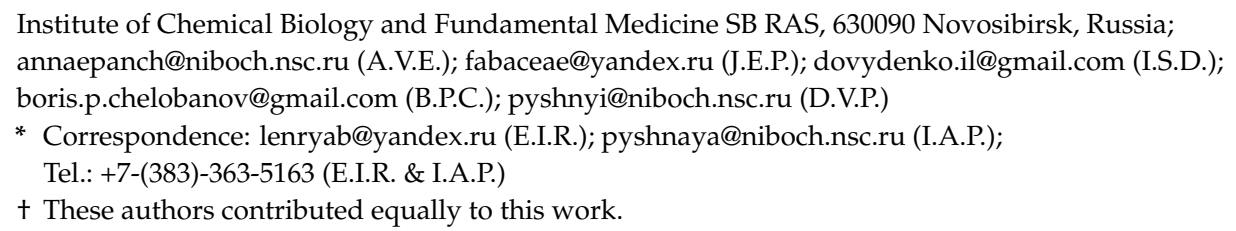

check for updates

Citation: Epanchintseva, A.V.; Poletaeva, J.E.; Dovydenko, I.S.; Chelobanov, B.P.; Pyshnyi, D.V.; Ryabchikova, E.I.; Pyshnaya, I.A. A Lipid-Coated Nanoconstruct Composed of Gold Nanoparticles Noncovalently Coated with Small Interfering RNA: Preparation, Purification and Characterization. Nanomaterials 2021, 11, 2775. https:// doi.org/10.3390/nano11112775

Academic Editor: Paulo Cesar De Morais

Received: 1 October 2021

Accepted: 18 October 2021

Published: 20 October 2021

Publisher's Note: MDPI stays neutral with regard to jurisdictional claims in published maps and institutional affiliations.

\begin{abstract}
There is an urgent need to develop systems for nucleic acid delivery, especially for the creation of effective therapeutics against various diseases. We have previously shown the feasibility of efficient delivery of small interfering RNA by means of gold nanoparticle-based multilayer nanoconstructs (MLNCs) for suppressing reporter protein synthesis. The present work is aimed at improving the quality of preparations of desired MLNCs, and for this purpose, optimal conditions for their multistep fabrication were found. All steps of this process and MLNC purification were verified using dynamic light scattering, transmission electron microscopy, and UV-Vis spectroscopy. Factors influencing the efficiency of nanocomposite assembly, colloidal stability, and purification quality were identified. These data made it possible to optimize the fabrication of target MLNCs bearing small interfering RNA and to substantially improve end product quality via an increase in its homogeneity and a decrease in the amount of incomplete nanoconstructs. We believe that the proposed approaches and methods will be useful for researchers working with lipid nanoconstructs.
\end{abstract}

Keywords: gold nanoparticles; siRNA; noncovalent adsorption; lipid enveloping; multilayer nanoconstructs for siRNA preparation and purification

\section{Introduction}

Nucleic-acid therapeutics have tremendous therapeutic potential for the treatment of many diseases. Nonetheless, the number of such therapeutics approved for clinical use is still low [1]. First of all, the reason is the nature of nucleic acids, which are macromolecules with a high negative charge and therefore are unable to penetrate into the cell on their own [2]. In addition, nucleic acids are very sensitive to nucleases, as evidenced by the low stability of nucleic acids in physiological fluids [3]. These obstacles can be overcome by (i) the development of modifications of nucleic acids and (ii) the creation of delivery systems that ensure not only penetration into the cell but also longer half-life of the nucleic acid formulation in the human body.

During the past several decades, a variety of systems of nucleic acid delivery have been proposed [4-8]. A distinct field of research and development of these systems is the use of solid (metal) nanoparticles enclosed in a shell and serving as a carrier of a nucleic acid. For instance, there are reports on the efficient delivery of small interfering RNA (siRNA) via gold nanoparticles (AuNPs) [9] and selenium nanoparticles [10] coated with chitosan and gold nanorods covered with two layers of polyelectrolytes [11].

Our study was aimed at creating multilayer nanoconstructs (MLNCs) based on spherical AuNPs on whose surface an siRNA layer is adsorbed and surrounded by a lipid 
envelope (Figure 1). The choice of AuNPs as the basis for this nanocomposite is due to their unique physicochemical properties and good biocompatibility [12-14].
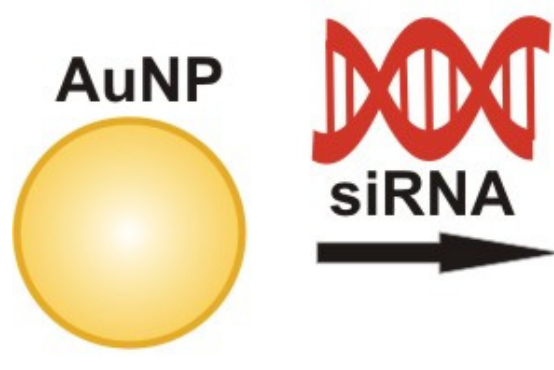

SIRNA
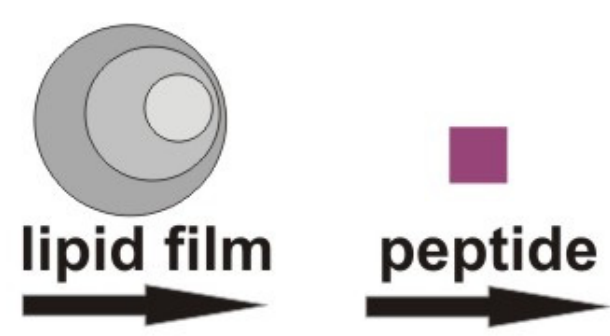

MLNC

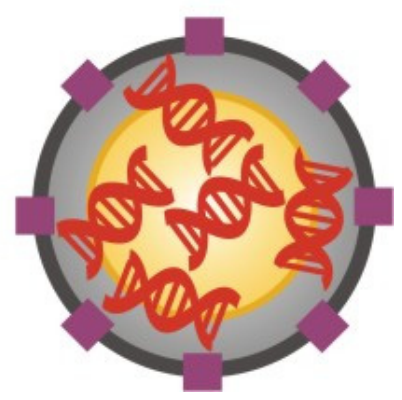

Figure 1. The fabrication and structure of the desired MLNC.

In the present study, the fabrication of an MLNC includes several steps: preparation of core nanoparticles (AuNP-siRNA) and a lipid film, assembly of the MLNC, and purification and concentration of the nanoconstruct particles. One of the previously developed versions of an MLNC-Whose lipid envelope is doped with peptide (LR) ${ }_{4} \mathrm{G}$ conjugated with stearic acid-Successfully delivers siRNA into cells and ensures a release of the siRNA cargo, as confirmed by the suppression of reporter protein expression (green fluorescent protein; GFP) [15]. In that study, we showed the feasibility of siRNA delivery via constructs containing lipid-coated AuNPs [15]. On the other hand, designing formulations intended for clinical use requires higher nanoconstruct quality, which can be achieved by improving the method of MLNC fabrication and will enhance the efficiency of siRNA delivery into human cells.

The second aim of this work was to optimize the conditions of MLNC synthesis that yield the most homogeneous suspensions free of empty lipid particles, naked core nanoparticles, and aggregates of core nanoparticles. To increase the efficiency of MLNC synthesis, we varied the conditions of all steps of this process on the basis of our previously obtained and published data about the stability and properties of nanoconstructs. In particular, we varied the ratio of core nanoparticles to a lipid mixture; an optimal buffer was chosen that enables effective wrapping of the core nanoparticles with a lipid envelope; and optimal conditions were found for the fractionation and purification of the MLNCs.

\section{Materials and Methods}

\subsection{Chemicals}

Tetrachloroauric acid trihydrate $\left(\mathrm{HAuCl}_{4} \cdot 3 \mathrm{H}_{2} \mathrm{O}\right)$ was purchased from Aurat (Moscow, Russia), and RNA phosphoramidites for oligoribonucleotide synthesis were acquired from Sigma-Aldrich (Hamburg, Germany). Sodium chloride $(\mathrm{NaCl})$ and magnesium sulfate $\left(\mathrm{MgSO}_{4}\right)$ were bought from Honeywell (Seelze, Germany) and sodium citrate dihydrate $\left(\mathrm{Na}_{3} \mathrm{C}_{6} \mathrm{H}_{5} \mathrm{O}_{7} \cdot 2 \mathrm{H}_{2} \mathrm{O}\right)$ from Fluka (Buchs, Switzerland). Magnesium acetate tetrahydrate $\left[\mathrm{Mg}\left(\mathrm{CH}_{3} \mathrm{COO}\right)_{2} \cdot 4 \mathrm{H}_{2} \mathrm{O}\right]$ was acquired from VWR International LLC (Radnor, PA, USA), whereas egg phosphatidylcholine and 1,2-dioleoyl-sn-glycero-3-phosphoethanolamine (DOPE) was obtained from Avanti (Alabaster, AL, USA). 2-[[4-Dodecylamino-6-oleylamino1,3,5-triazine-2yl]-(2-hydroxyethyl)amino]ethanol (DOME2) was synthetized as described in ref. [13]. Disodium phosphate dihydrate $\left(\mathrm{NaH}_{2} \mathrm{PO}_{4} \cdot 2 \mathrm{H}_{2} \mathrm{O}\right)$ and monosodium phosphate dodecahydrate $\left(\mathrm{Na}_{2} \mathrm{HPO}_{4} \cdot 12 \mathrm{H}_{2} \mathrm{O}\right)$ were purchased from Reatex (Moscow, Russia) and uranyl acetate from SPI (West Chester, PA, USA). Peptide $\mathrm{NH}_{2}-(\mathrm{RL})_{4} \mathrm{G}-\mathrm{C}(\mathrm{O}) \mathrm{NH}_{2}$. $5 \mathrm{CF}_{3} \mathrm{COOH}$ was acquired from Diapharm (Lyubertsy, Russia). Sodium acetate trihydrate $\left(\mathrm{CH}_{3} \mathrm{COONa} \cdot 3 \mathrm{H}_{2} \mathrm{O}\right)$, acetic acid $\left(\mathrm{CH}_{3} \mathrm{COOH}\right)$, and sucrose $\left(\mathrm{C}_{12} \mathrm{H}_{22} \mathrm{O}_{11}\right)$ were bought from Panreac (Barcelona, Spain), whereas cesium chloride $(\mathrm{CsCl})$ and glycerol $\left(\mathrm{C}_{3} \mathrm{H}_{8} \mathrm{O}_{3}\right)$ were ob- 
tained from Applichem (Darmstadt, Germany), and trichloromethane (chloroform, $\mathrm{CHCl}_{3}$ ) and methanol $\left(\mathrm{CH}_{3} \mathrm{OH}\right)$ were purchased from Reachem (Moscow, Russia). AlamarBlue ${ }^{\mathrm{TM}}$ Cell Viability Reagent was acquired from Invitrogen (Waltham, MA, USA). Water was purified by means of a Simplicity 185 water purification system (Millipore, Burlington, MA, USA) and had a resistivity of $18.2 \mathrm{M} \Omega \cdot \mathrm{cm}$ at $25^{\circ} \mathrm{C}$.

\subsection{Preparation of Core Nanoparticles}

To obtain core nanoparticles (AuNP-siRNA), siRNA was used that suppresses GFP synthesis in cultured cells stably expressing this protein. The sequence of the siRNA was as follows: sense strand, 5'-CAAGCUGACCCUGAAGUUCTT; and antisense strand, 5' GAACUUCAGGGUCAGCUUGTT [16]. The synthesis of this siRNA is described in detail in our previous work [17]. The AuNPs were synthesized using a previously published technique [18].

The core nanoparticles, which are AuNPs noncovalently covered with siRNA, were fabricated as described elsewhere [15,19], with some modifications: 4 mM citrate-stabilized AuNPs (size $12 \pm 1 \mathrm{~nm}$ according to transmission electron microscopy [TEM] or $17.3 \pm 2.1 \mathrm{~nm}$ according to dynamic light scattering [DLS] analysis; a zeta potential of $-33.6 \pm 2.0 \mathrm{mV}$ according to DLS) at a concentration of $3.6 \times 10^{-9} \mathrm{M}$ were incubated with $0.72 \mu \mathrm{M}$ siRNA for $22 \mathrm{~h}$ at room temperature in the presence of either 5 or $10 \mathrm{mM} \mathrm{NaCl}$ and either $\mathrm{Mg}\left(\mathrm{CH}_{3} \mathrm{COO}\right)_{2}$ or $\mathrm{MgSO}_{4}$. The resultant suspensions of core nanoparticles were centrifuged, the supernatant was discarded, and the pellet containing the core nanoparticles was washed with $1 \mathrm{~mL}$ of $4 \mathrm{mM}$ sodium citrate dihydrate. The size and monodispersity of the obtained core nanoparticles were determined using TEM (13 $\pm 1 \mathrm{~nm})$ and DLS $(25 \pm 9 \mathrm{~nm})$. The zeta potential of the core nanoparticles proved to be $-44 \pm 1 \mathrm{mV}$, and the polydispersity index (PDI) of the suspension was $0.212 \pm 0.011$. The core nanoparticles had a surface plasmon resonance maximum at $520 \mathrm{~nm}$, and the density of siRNA molecules on the AuNP surface was $29 \pm 6$.

\subsection{Preparation of a Lipid Film}

The lipid film was prepared as described before [15], with modifications: $90 \mu \mathrm{L}$ of $1 \mathrm{mM}$ egg phosphatidylcholine and DOPE in a $\mathrm{CHCl}_{3} / \mathrm{CH}_{3} \mathrm{OH}$ mixture $(1: 1)$ and $10 \mu \mathrm{L}$ of $1 \mathrm{mM}$ DOME2 in $\mathrm{CHCl}_{3}$ were added to $1 \mathrm{~mL}$ of $\mathrm{CHCl}_{3}$ in a $10 \mathrm{~mL}$ round-bottom flask. Next, the solvent was evaporated either at $25^{\circ} \mathrm{C}$ and $6 \mathrm{mmHg}$ or without thermostatting at $12 \mathrm{mmHg}$. The resultant lipid film was then dried in vacuum in a desiccator to remove traces of the organic solvents, after which the flask with the film was incubated at $-18{ }^{\circ} \mathrm{C}$ for $16 \mathrm{~h}$. All the procedures for obtaining the lipid film were carried out in an argon atmosphere.

\subsection{Synthesis of a Peptide Conjugate with Stearic Acid}

A conjugate of a peptide $\left[(\mathrm{RL})_{4} \mathrm{G}-\mathrm{NH}_{2}\right.$ ] with stearic acid [Str-(RL) $\left.{ }_{4} \mathrm{G}-\mathrm{NH}_{2}\right]$ for doping the lipid envelope was synthesized as described previously [15].

\subsection{Assembly of the MLNC}

The procedure was performed as described earlier [15], with slightly modified reaction conditions, as described below. The MLNCs were obtained in two stages:

First, a buffer with $\mathrm{pH} 4.5$ was applied to the surface of the lipid film: either (1) $0.9 \mathrm{~mL}$ of $\mathrm{H}_{2} \mathrm{O}$ and $31 \mu \mathrm{L}$ of $\mathrm{NaH}_{2} \mathrm{PO}_{4}(1.00 \mathrm{M}, 0.10 \mathrm{M}$, or $0.01 \mathrm{M})$ or (2) $0.825 \mathrm{~mL}$ of $\mathrm{H}_{2} \mathrm{O}$; to the buffer, we added $100 \mu \mathrm{L}$ of a suspension of the core nanoparticles $(2.5 \mathrm{pmol}$ in terms of gold) in $6 \mathrm{mM} \mathrm{CH}_{3} \mathrm{COOH}$. The reaction mixture of the core nanoparticles and lipid film was sonicated at $90 \mathrm{~W}$ for $15 \mathrm{~min}$ at $25^{\circ} \mathrm{C}$. The expected result of this step is a suspension of the core nanoparticles bearing a layer of noncovalently attached siRNA enclosed in a lipid envelope.

Second stage: the $\mathrm{pH}$ of the suspension containing the core nanoparticles carrying the lipid envelope was adjusted to 7.4 by the addition of either (1) $69 \mu \mathrm{L}$ of $1.00,0.10$, or $0.01 \mathrm{M}$ 
$\mathrm{Na}_{2} \mathrm{HPO}_{4}$ or (2) $100 \mu \mathrm{L}$ of $3 \mathrm{mM} \mathrm{CH} \mathrm{COONa}_{3}$. Then, $10 \mu \mathrm{L}$ of $1 \mathrm{mM}$ stearic-acid-conjugated peptide, Str-(LR) $)_{4} \mathrm{G}$, was introduced. The reaction mixture was sonicated $(90 \mathrm{~W})$ for $5 \mathrm{~min}$ at $25^{\circ} \mathrm{C}$. The size and monodispersity of the obtained MLNCs were verified using TEM and DLS. The MLNCs had a surface plasmon resonance maximum at $534 \mathrm{~nm}$.

Thus, we obtained a suspension of MLNCs whose envelope was doped with a peptide. For brevity, hereafter, these nanoparticles are referred to as "MLNCs," without a mention of the peptide.

\subsection{Purification of the MLNCs by Banding Centrifugation}

Fractionation of the MLNCs was carried out either $\{1\}$ in a cesium chloride gradient or $\{2\}$ in solutions of glycerol or sucrose.

$\{1\} \mathrm{In}$ a $1.5 \mathrm{~mL}$ test tube, we sequentially layered $500 \mu \mathrm{L}$ each of an aqueous $61.7 \%$ $\mathrm{CsCl}$ solution $\left(\rho=1.789 \mathrm{~g} / \mathrm{cm}^{3}, \mu=1.2389 \mathrm{cP}\right)$ and an aqueous $10.8 \% \mathrm{CsCl}$ solution $\left(\rho=1.0804 \mathrm{~g} / \mathrm{cm}^{3}, \mu=0.966 \mathrm{cP}\right)$ and then $50 \mu \mathrm{L}$ of the MLNC suspension; its colloidal stability was examined by means of changes in the color of the MLNC layer.

\{2\} In a $15 \mathrm{~mL}$ test tube, we layered 500-1000 $\mu \mathrm{L}$ of the MLNC suspension on top of $10 \mathrm{~mL}$ of (i) an aqueous $75 \%$ glycerol solution $(\mu=41 \mathrm{cP}$ ) or (ii) an aqueous $26 \%$ sucrose solution in $1 \mathrm{mM}$ phosphate buffer ( $\mathrm{pH}$ was altered) $(\rho=1.1082 \mathrm{~g} / \mathrm{mL}, \mu=2.223 \mathrm{cP})$ or (iii) an aqueous $58 \%$ sucrose solution in $1 \mathrm{mM}$ phosphate buffer $(\rho=1.267 \mathrm{~g} / \mathrm{mL}$, $\mu=42.8 \mathrm{cP}$ ), followed by centrifugation at $25^{\circ} \mathrm{C}$ in the range of $1000-8000 \times g$ for $15-90 \mathrm{~min}$. Fractions of different colors were collected into separate $1.5 \mathrm{~mL}$ tubes and were subjected to purification to remove excess glycerol or sucrose via dialysis or centrifugation. After that, all the fractions were analyzed using DLS and TEM.

After the fractionation in sucrose, the samples with or without an added equal volume of $1 \mathrm{mM}$ phosphate buffer were centrifuged in $1.5 \mathrm{~mL}$ tubes at $2000 \times \mathrm{g}$ for 15,30 , or $60 \mathrm{~min}$, the supernatant was discarded, and the resulting MLNCs were analyzed via DLS, TEM, and UV-Vis spectroscopy.

After the fractionation in glycerol, dialysis was performed either in Centricon Plus-70 $3 \mathrm{kDa}$, Merck KGaA (Darmstadt, Germany) or in screw cap $1.5 \mathrm{~mL}$ tubes at $25{ }^{\circ} \mathrm{C}$ for 16-72 $\mathrm{h}$ against 1,3 , or $10 \mathrm{mM}$ phosphate buffer using either (1) membranes for extruders with a pore diameter of 30 or $100 \mathrm{~nm}$, Whatman (Maidstone, UK) or (2) single-layer 3.5 or $10 \mathrm{kDa}$ dialysis bags, Thermo Fisher Scientific (Göteborg, Sweden).

\subsection{Examination of the Composition and Quality of the Samples}

\subsubsection{Optical Extinction Spectra}

To verify colloidal stability and to determine gold concentration, we performed UV-Vis spectroscopy. Optical adsorption spectra of the core nanoparticles and all preparations of MLNCs (Figure 2) were recorded on a Clariostar plate fluorimeter, BMG Labtech (Ortenberg, Germany) in the range $400-800 \mathrm{~nm}$ according to the manufacturer's instructions. The absorption maximum of all versions of MLNC preparations was found to be shifted by $12 \mathrm{~nm}$ relative to AuNPs and core nanoparticles; this shift emerged after the lipid envelope formation and was not affected by other experimental conditions. Preservation of colloidal stability of the samples during the procedure was confirmed by the absence of a peak in the range $600-700 \mathrm{~nm}$. 


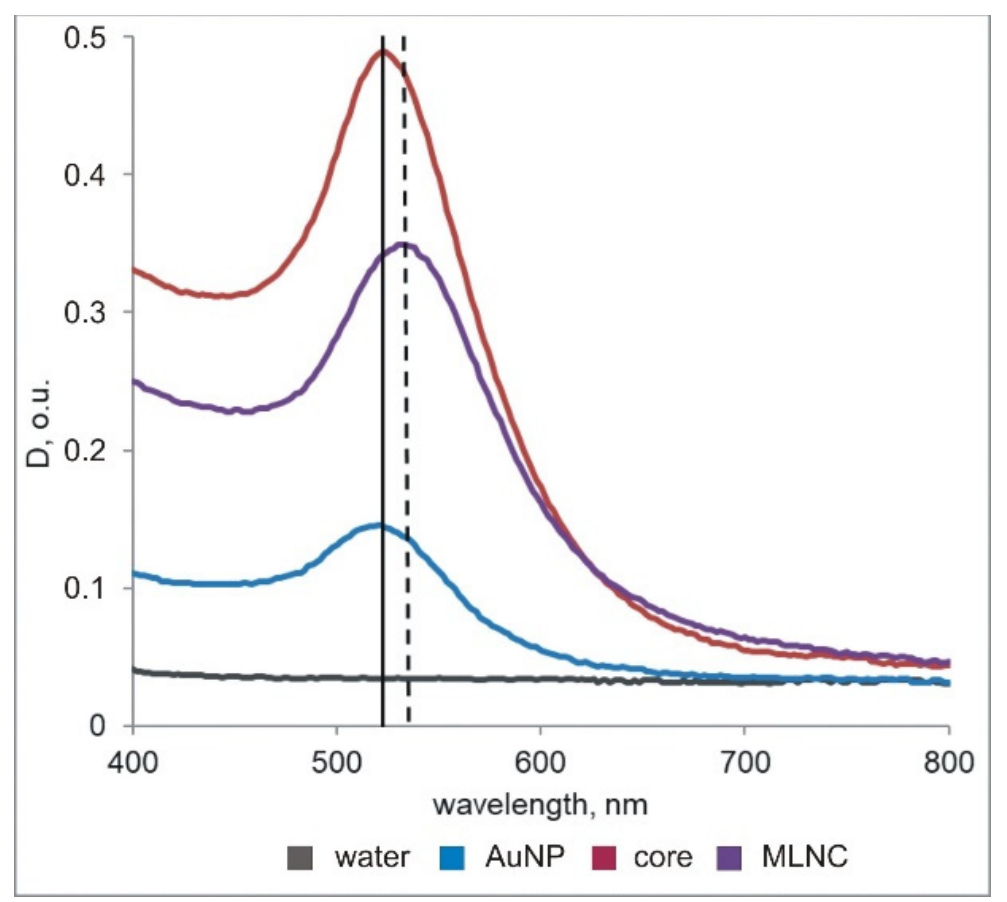

Figure 2. "Typical" spectra of the obtained samples of AuNPs, core nanoparticles, and MLNCs. The vertical lines indicate absorption maxima for AuNPs and core nanoparticles $(520 \mathrm{~nm}$, left-hand line) and for MLNCs (532 nm, right-hand line). For clarity, we present the spectra of the samples at concentrations that ensure resolution of the lines in the graph.

The yield of MLNCs was calculated from optical density of the final suspension at $520 \mathrm{~nm}$ using the molar extinction coefficient characteristic of the original AuNPs $\left(\varepsilon=8.78 \times 10^{8} \mathrm{~L} \cdot \mathrm{mol} / \mathrm{cm}\right)$ [20]. The amount (mol) of AuNPs used to fabricate the core nanoparticles was set to $100 \%$.

\subsubsection{DLS}

All samples of core nanoparticles and MLNCs were characterized via DLS and TEM. DLS analysis is employed for assessing overall hydrodynamic characteristics of a suspension of nanoparticles, whereas TEM provides information on the fine structure of nanoparticles and its changes. These methods complement each other and, taken together, help to exhaustively characterize samples of nanoconstructs.

Suspensions of the core nanoparticles and MLNCs were analyzed using DLS by means of a Malvern Zetasizer Nano instrument, Malvern Instruments (Worcestershire, UK) in accordance with the manufacturer's instructions. This method allowed us to characterize particle suspensions in terms of the following parameters: the hydrodynamic diameter,

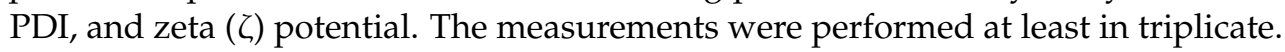

\subsubsection{TEM}

For examination using TEM, $10 \mu \mathrm{L}$ of a suspension of the core nanoparticles or MLNCs was applied to a formvar film on a grid and incubated for $1 \mathrm{~min}$. Then, the drop was aspirated with a pipettor, and without drying, the grid was placed on a drop of uranyl acetate for $10 \mathrm{~s}$; the excess liquid was removed with filter paper. All TEM samples were prepared identically; at least five grid cells were examined in different parts of each grid. The suspensions of the core nanoparticles and MLNCs were investigated under a JEM 1400 transmission electron microscope, Jeol (Tokyo, Japan) equipped with a Veleta digital camera, EM SIS (Muenster Germany). Particle sizes were determined using iTEM software, version 5.2, EM SIS (Muenster, Germany). 


\subsection{Cytotoxicity Assays of Glycerol and Sucrose}

To this end, SC-1 R780 fibroblasts were cultured in the IMDM medium, Gibco (Grand Island, NY, USA) supplemented with $10 \%$ of fetal calf serum (Gibco), penicillin $(100 \mathrm{U} / \mathrm{mL})$, and streptomycin $(100 \mu \mathrm{g} / \mathrm{mL})$ in a humidified atmosphere containing $5 \%$ of $\mathrm{CO}_{2}$ at $37^{\circ} \mathrm{C}$. The cytotoxicity assay was performed using the AlamarBlue test [21].

\subsection{Statistical Analysis}

Each experiment was conducted independently at least three times. Data are presented as mean \pm standard deviation from at least three independent experiments.

\section{Results}

\subsection{The Mechanism of MLNC Formation}

The formation of a lipid envelope around the core nanoparticle is mediated by electrostatic interaction between protonated amino groups of the lipid (DOME2, which is a component of the lipid film) and negatively charged phosphate groups of the siRNA on the surface of the core nanoparticle. Because DOME2 is protonated at a $\mathrm{pH}$ below 7, the assembly of the nanoconstructs was carried out in an acidic medium. This strategy necessitated selecting a buffer that makes it easy to switch DOME2 to either a charged (protonated) state or a neutral (deprotonated) state and ensures colloidal stability of the core nanoparticles. We believe that choosing such a buffer will reduce the aggregation of MLNC particles and aggregation of "naked" core nanoparticles.

To form a lipid envelope around the core nanoparticle, a suspension of the core nanoparticles in an acidic buffer was applied to the prepared lipid film, and the reaction mixture was sonicated (Figure 3A). The lipid film, which carries the core nanoparticles adsorbed on the surface, gets fragmented, and the core nanoparticles end up in a lipid envelope. The sizes of the forming particles are different, as are the numbers of core nanoparticles enclosed in a single shell. Consequently, the resulting suspension contains structurally diverse desired MLNCs ("target MLNCs": Figure 3B, panel 1), aggregates of the core nanoparticles surrounded by a thin lipid envelope (Figure 3B, panel 2), lipid particles and empty vesicles (Figure 3B, panel 3), lipid film fragments of various sizes with adhered core nanoparticles (Figure 3B, panel 4), and naked core nanoparticles, both stand-alone and aggregated (Figure 3B, panel 5).

It is obvious that the ideal end product should consist mainly of stand-alone MLNCs each containing one core nanoparticle. The presented mechanism of MLNC formation makes this outcome a pipe dream, mainly owing to the heterogeneity of adsorption of the core nanoparticles on the lipid film. Therefore, our efforts were focused on obtaining suspensions with the highest proportion of stand-alone MLNCs containing 1-10 core nanoparticles and a distinct electron-transparent envelope; such nanoconstructs are hereinafter referred to as "target MLNCs" (Figure 3B, panel 1). Meanwhile, we strived to reduce the concentrations of empty lipid particles, aggregates of core nanoparticles enclosed in a thin lipid envelope, and naked (unenveloped) core nanoparticles (Figure 3B, panels 2-5). It should be noted that we always assessed the effect of one or another modification of the protocol by means of changes in the characteristics of the end product: the final suspension of MLNCs. 


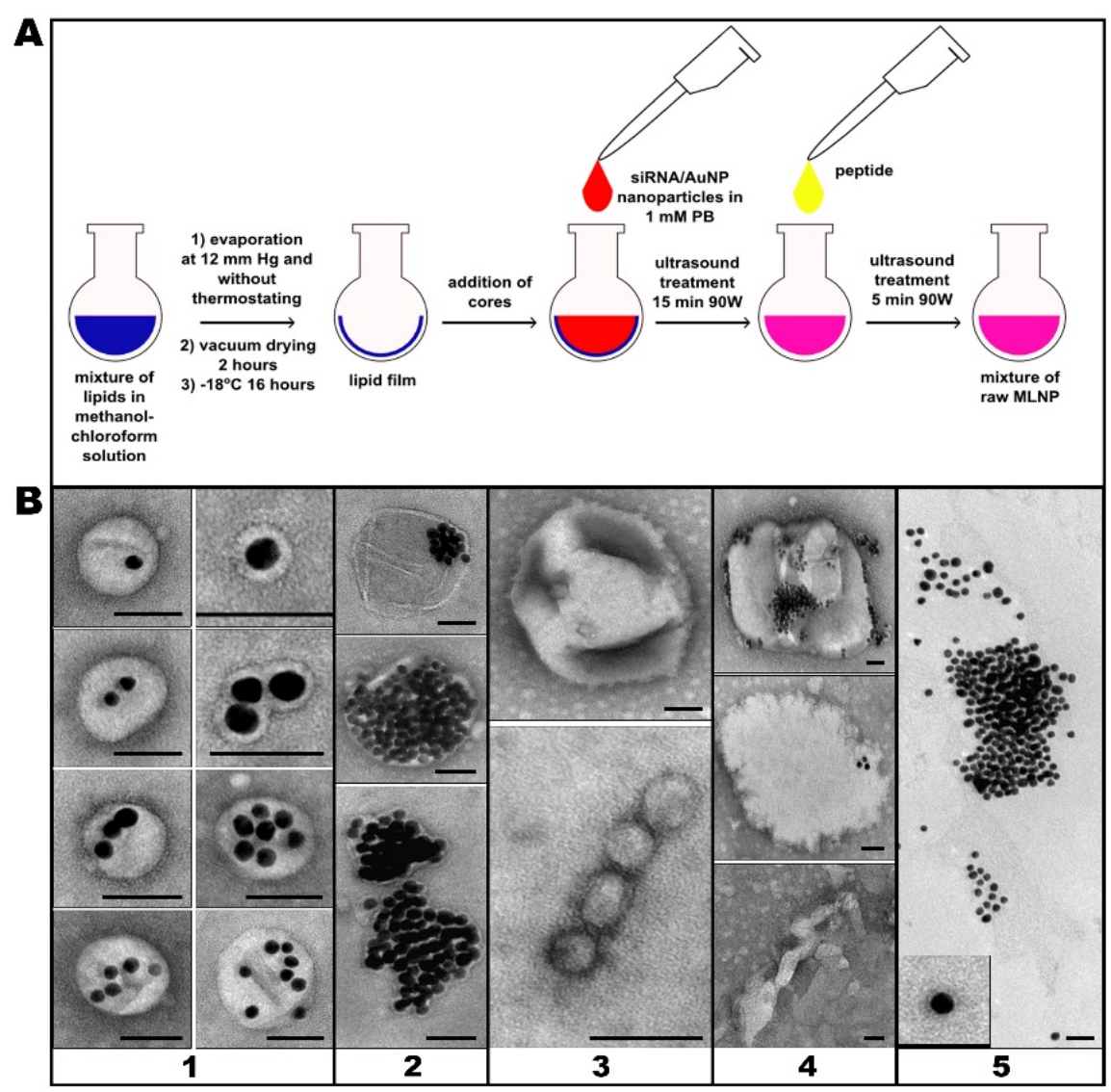

Figure 3. (A) The scheme of MLNC formation after the application of a suspension of the core nanoparticles to the lipid film and sonication. (B) Morphology of the particles in the MLNC preparations. Panel 1: Different subtypes of a "target MLNC" containing 1 to 10 electron-dense core nanoparticles; Panel 2: Aggregates of the core nanoparticles surrounded by a thin lipid envelope; Panel 3: Empty lipid particles; Panel 4: Fragments of the lipid film with adhered core nanoparticles; Panel 5: "Naked" core nanoparticles. The scale bars correspond to $50 \mathrm{~nm}$. Negative staining with uranyl acetate followed by TEM.

\subsection{Assembly of the Core Nanoparticles: AuNP-siRNA}

The first step in the fabrication of MLNCs is the synthesis of core nanoparticles (AuNPsiRNA); we have described this procedure in detail previously $[15,22,23]$. For enveloping the core nanoparticles with the siRNA, a suitable buffer was experimentally selected, phosphate buffer, which enabled easy switching from $\mathrm{pH} 5$ to $\mathrm{pH}$ 7.4, which corresponds to physiological $\mathrm{pH}$. It is important that this buffer ensured colloidal stability of the core nanoparticles throughout the entire experiment on MLNC fabrication.

During the preparation of MLNCs, it became apparent that the lipid particles, which contained lipid DOME2, had negative $\zeta$ potential in acidic phosphate buffer, indicating adsorption of phosphate anions on the surface of the lipid film. Accordingly, the phosphate component of the buffer will compete with the core nanoparticles during their adsorption onto the lipid film, thereby inevitably decreasing the efficiency of the enveloping process. At first glance, the simplest solution seems to be to change the buffer, but this approach can diminish colloidal stability of the core nanoparticles. We reduced the phosphate anion concentration from 0.100 to $0.001 \mathrm{M}$, which increased the efficiency of MLNC formation (Figure 4) and decreased the PDI (Table 1). 

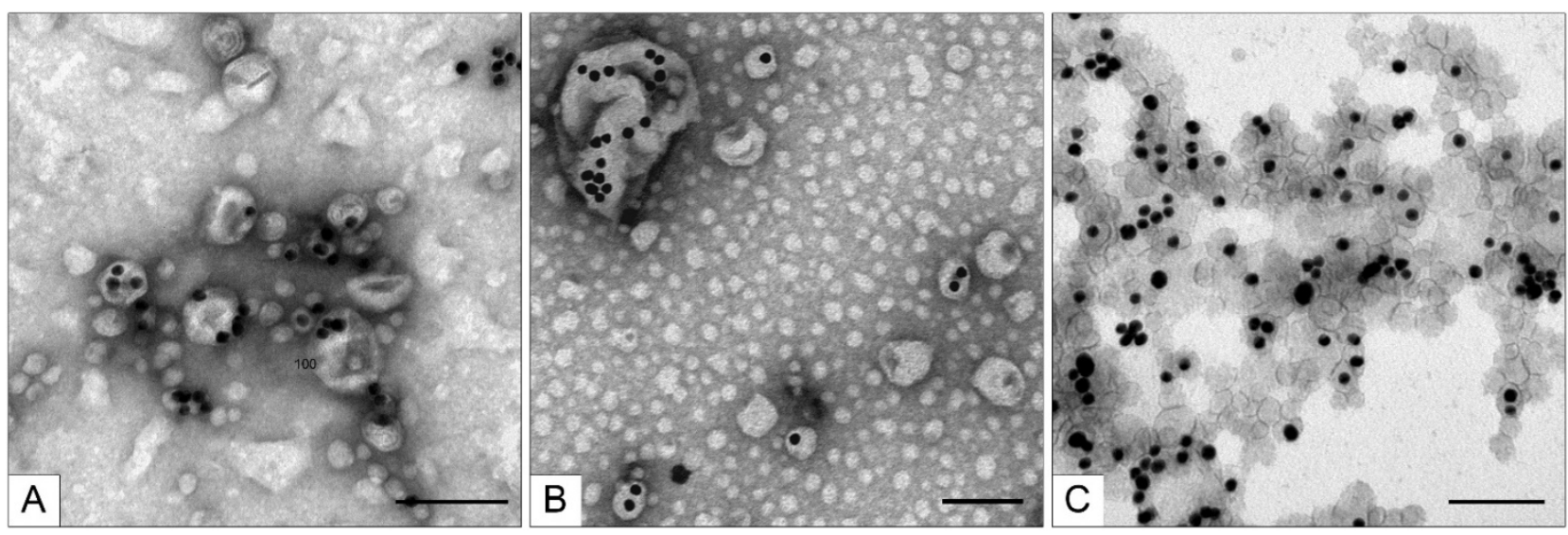

Figure 4. Ultrastructure of the obtained MLNCs. (A) Assembly in $100 \mathrm{mM}$ phosphate buffer, (B) assembly in $10 \mathrm{mM}$ phosphate buffer, and (C) assembly in $1 \mathrm{mM}$ phosphate buffer. The scale bars correspond to $100 \mathrm{~nm}$. Negative staining with a $0.5 \%$ uranyl acetate solution followed by TEM.

Table 1. Physicochemical parameters of MLNCs at different concentrations of phosphate buffer (PB).

\begin{tabular}{cccc}
\hline MLNC Sample ID & PB Concentration, $\mathbf{m M}$ & PDI & Hydrodynamic Diameter, $\mathbf{n m}$ \\
\hline 1 & 100 & $0.203 \pm 0.012$ & $749.2 \pm 177$ \\
2 & 10 & $0.256 \pm 0.010$ & $169 \pm 88$ \\
3 & 3 & $0.275 \pm 0.015$ & $137.7 \pm 65$ \\
4 & 1 & $0.209 \pm 0.009$ & $158.7 \pm 74$ \\
\hline
\end{tabular}

By lowering the buffer concentration, we reduced the amount of phosphate anions adsorbed on the lipid film surface; however, it was necessary to neutralize the effect of the remaining ones. For this purpose, it was decided to add a divalent cation that would act as a linker between the phosphate anions adsorbed on the lipid film surface and the siRNA on the surface of AuNPs. $\mathrm{Mg}^{2+}$ was chosen and did not lead to "adhesion" and precipitation of the components of the lipid film and core nanoparticles, nor does it catalyze phosphodiester bond hydrolysis in siRNA. Nevertheless, which salt can be used in this context? It is known that the type of anion has a pronounced effect on colloidal stability of core nanoparticles [24-26]. Accordingly, we introduced either magnesium acetate or magnesium sulfate into the reaction mixture.

During the synthesis of the core nanoparticles, the influence of $\mathrm{Mg}\left(\mathrm{CH}_{3} \mathrm{COO}\right)_{2}$ or $\mathrm{MgSO}_{4}$ at 0.1 or $0.4 \mathrm{mM}$ added to the reaction mixture was determined by means of physicochemical characteristics (Table 2) and alterations of colloidal stability of the core nanoparticle suspension. Acetate ions at both concentrations affected the colloidal stability negatively. The addition of magnesium ions in the form of $0.1 \mathrm{mM}$ sulfate "improved" hydrodynamic parameters of the core nanoparticles and did not alter colloidal stability for several days. At $0.4 \mathrm{mM}$, the impact of this salt was negative.

Table 2. Physicochemical characteristics of the core nanoparticles in phosphate buffer containing different magnesium salts.

\begin{tabular}{ccccc}
\hline Sample & Magnesium Salt & $\zeta$ Potential, mV & Hydrodynamic Diameter, nm & PDI \\
\hline Core nanoparticles & none & $-44 \pm 1$ & $25 \pm 9$ & $0.212 \pm 0.011$ \\
Core nanoparticles & $0.1{\mathrm{mM} \mathrm{Mg}(\mathrm{Ac})_{2}}$ & $-45 \pm 1$ & $27 \pm 11$ & $0.416 \pm 0.041$ \\
Core nanoparticles & $0.1 \mathrm{mM} \mathrm{MgSO}_{4}$ & $-39 \pm 1$ & $25 \pm 9$ & $0.266 \pm 0.050$ \\
Core nanoparticles & $0.4 \mathrm{mM} \mathrm{MgSO}_{4}$ & $-40 \pm 2$ & $90 \pm 85$ & $0.565 \pm 0.316$ \\
\hline
\end{tabular}

Having found the optimal concentration of $\mathrm{MgSO}_{4}(0.1 \mathrm{mM})$ for obtaining the core nanoparticles, we began to vary the concentration of $\mathrm{NaCl}$, the presence of which is nec- 
essary for denser loading of siRNA molecules onto AuNPs. AuNPs are very sensitive to monovalent anions [25], and even a small shift in their concentration can affect colloidal stability of the resulting core nanoparticles. Previously [15], we prepared core nanoparticles at $10 \mathrm{mM} \mathrm{NaCl}$ (parameters of the obtained preparations of MLNCs: hydrodynamic diameter of $205 \pm 100 \mathrm{~nm}$ and PDI of 0.186), but in the present work, we reduced the $\mathrm{NaCl}$ concentration in half, which increased suspension homogeneity and reduced the size of MLNCs (hydrodynamic diameter became $128 \pm 54 \mathrm{~nm}$, and PDI became 0.151). The change in $\mathrm{NaCl}$ concentration did not lead to a noticeable alteration of the end products' composition according to TEM; they contained MLNCs with different numbers of core nanoparticles as well as lipid particles, empty lipid vesicles, aggregates of the core nanoparticles, and a small percentage of naked core nanoparticles (Figure 5). In subsequent experiments, the reaction mixture version containing $0.1 \mathrm{mM} \mathrm{MgSO}_{4}$ and $5 \mathrm{mM} \mathrm{NaCl}$ was utilized to synthesize the core nanoparticles.
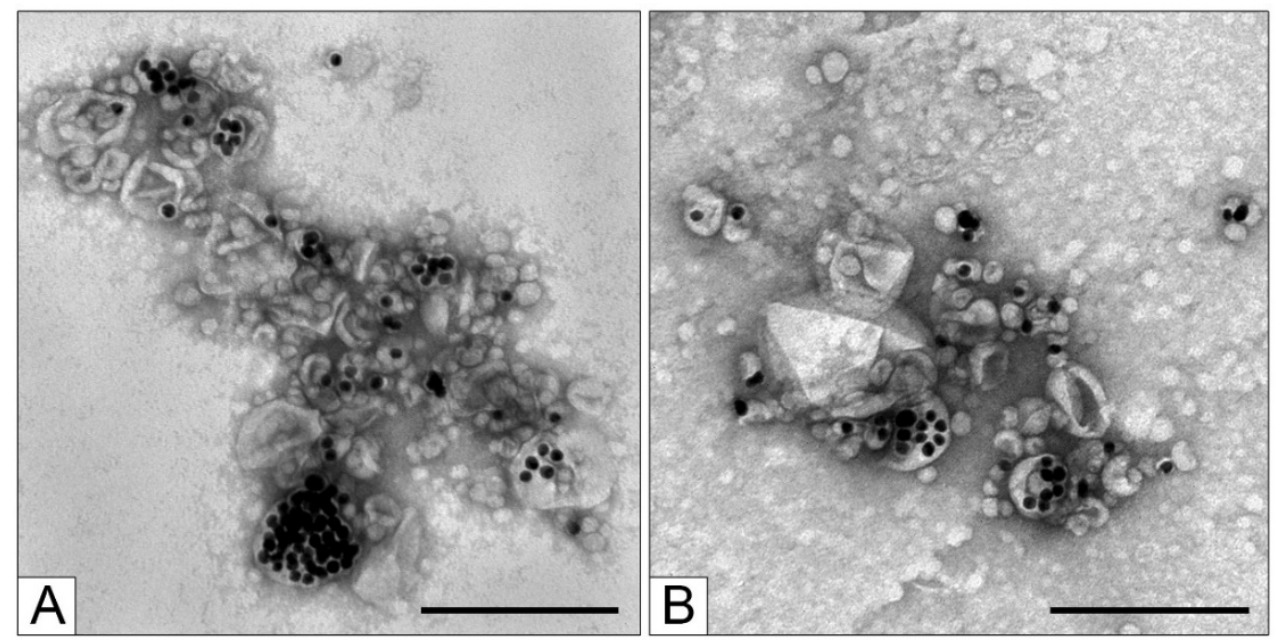

Figure 5. Ultrastructure of the MLNCs obtained in the presence of $0.1 \mathrm{mM} \mathrm{MgSO}_{4}$. (A) MLNCs prepared via the addition of $10 \mathrm{mM} \mathrm{NaCl}$ or (B) $5 \mathrm{mM} \mathrm{NaCl}$. The scale bars correspond to $200 \mathrm{~nm}$. Negative staining with a $0.5 \%$ uranyl acetate solution followed by TEM.

\subsection{Fabrication of the Lipid Film}

The lipid film was generated via evaporation of a solution of mixed lipids in a chloroform/methanol mixture under reduced pressure $(6 \mathrm{mmHg})$. We found that the evaporation rate, which can be adjusted by changing pressure, affects the quality of the resulting film: an increase in pressure from 6 to $12 \mathrm{mmHg}$ and elimination of flask thermostatting improved the homogeneity of the lipid film and facilitated its interaction with the core nanoparticles. Accordingly, more homogeneous end products with a higher concentration of MLNCs were obtained. For instance, the hydrodynamic diameter of MLNCs decreased from $188 \pm 96$ to $144.3 \pm 79 \mathrm{~nm}$, while the PDI was $0.259 \pm 0.005 \mathrm{~nm}$ and $0.250 \pm 0.006 \mathrm{~nm}$, respectively. TEM analysis of these samples revealed no appreciable differences in their composition: they contained MLNCs of various structures, empty lipid particles, aggregates of the core nanoparticles within a thin lipid envelope, and naked core nanoparticles (Figure 6). 


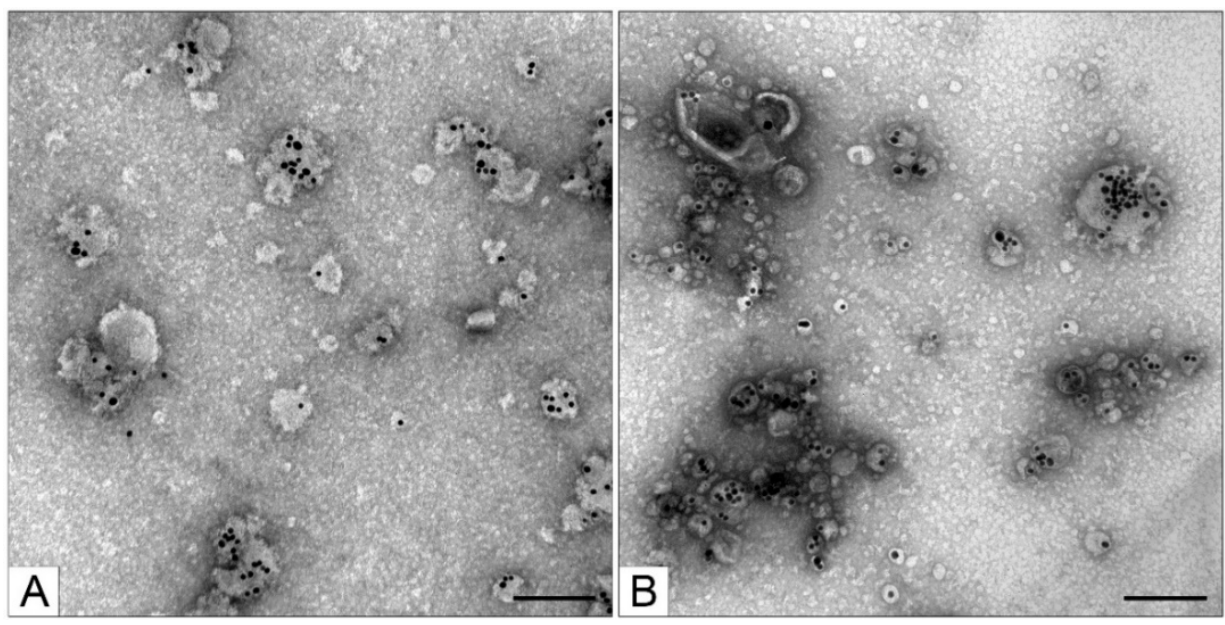

Figure 6. Ultrastructure of the MLNCs prepared from the lipid film synthesized under a pressure of $12 \mathrm{mmHg}$ (A) or $6 \mathrm{mmHg}$ (B). The scale bars correspond to $200 \mathrm{~nm}$. Negative staining with a $0.5 \%$ uranyl acetate solution followed by TEM.

\subsection{Doping of the Lipid Envelope of MLNCs with the Peptide}

The final step in the fabrication of the target MLNCs is doping of the lipid envelope with peptide Str-(LR) ${ }_{4} \mathrm{G}$ at $\mathrm{pH} 7.4$ to make sure that the nanoparticles can penetrate into the cell and to overcome endosomal arrest as well as to enable the release of the siRNA into the cytosol from the surface of the core nanoparticle. The effectiveness of this procedure has been demonstrated by us previously [15]. In the present work, a sample of lipid-coated core nanoparticles obtained at $\mathrm{pH} 4.5$ via sonication was transferred to a medium with $\mathrm{pH}$ 7.5 , after which the peptide was added, and the mixture was sonicated for $5 \mathrm{~min}$ at $25^{\circ} \mathrm{C}$.

It turned out that the doping of the lipid envelope with Str-(LR) ${ }_{4} \mathrm{G}$ affects physicochemical parameters of the resultant MLNCs: the addition of the peptide yielded an increase in the hydrodynamic diameter from $118.4 \pm 44.27$ to $235.1 \pm 100.3 \mathrm{~nm}$, while the PDI was 0.1942 and 0.1854 for the MLNCs without doping and after the doping, respectively. Structural analysis of the end product using TEM did not uncover any noticeable changes in its composition and in particle structure of the MLNCs after the doping with peptide Str-(LR) ${ }_{4} \mathrm{G}$.

These findings allowed us to find optimal modifications of the initial protocol [15] that afford the best quality of MLNCs: the presence of $0.1 \mathrm{mM} \mathrm{MgSO}_{4}$ and $5 \mathrm{mM} \mathrm{NaCl}$ during the assembly of the core nanoparticles, lipid film synthesis at $12 \mathrm{mmHg}$ without thermostatting, and the assembly of MLNCs in $1 \mathrm{mM}$ phosphate buffer. The end product obtained under these conditions is hereinafter referred to as "optimized MLNCs" (Figure 7). The preparations of optimized MLNCs are characterized by a hydrodynamic diameter of $152 \pm 75 \mathrm{~nm}$ and a PDI of $0.201 \pm 0.012$.

The presented modifications of the protocol of MLNC fabrication increased the efficiency of synthesis of core nanoparticles carrying the lipid envelope, but the obtained suspensions still contained a noticeable amount of lipid particles and vesicles, aggregates of the core nanoparticles within a thin lipid envelope, and naked core nanoparticles, thus necessitating purification of the MLNC preparations. Given that the MLNCs, lipid particles, and core nanoparticles have different densities and sizes, they can be separated using banding high-speed centrifugation. 


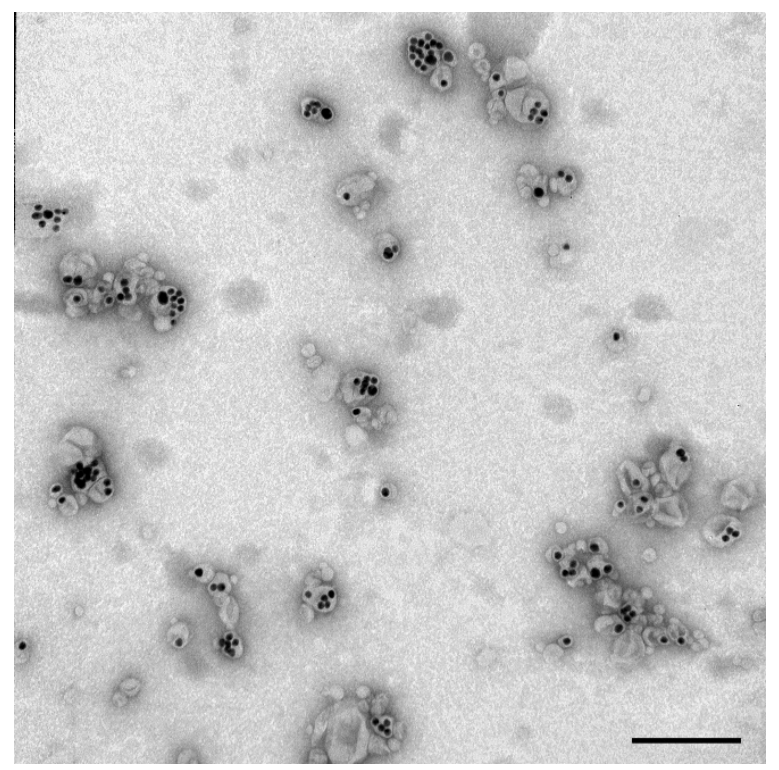

Figure 7. Ultrastructure of optimized MLNCs. The scale bar corresponds to $200 \mathrm{~nm}$. Negative staining with a $0.5 \%$ uranyl acetate solution followed by TEM.

\subsection{Fractionation of MLNCs via Centrifugation}

Cesium chloride density gradient fractionation is the best-studied and widely used technique for the separation of macromolecules. We fractionated optimized MLNCs in a stepwise $\mathrm{CsCl}$ gradient; as a consequence, the color of the sample turned from dark red to blue, indicating complete loss of colloidal stability of these nanoparticles in the cesium chloride solution.

After giving up on cesium chloride, for the fractionation of MLNCs, we chose an aqueous solution of sucrose as a viscous and less "aggressive" medium. The fractionation was conducted in a homogeneous solution without a concentration gradient. The sucrose concentration was selected according to calculations suggesting that target MLNCs should penetrate into the viscous medium to $2 \mathrm{~cm}$ depth. The computation was performed using a calculator [27]. To this end, the density of the nanoparticles was assumed to equal that of gold, $19 \mathrm{~g} / \mathrm{cm}^{3}$, and we hypothesized that during the centrifugation, the less dense envelope and the heavy dense core would move at different acceleration rates. In accordance with the calculation results, a $58 \%$ sucrose solution was selected $(\rho=1.267 \mathrm{~g} / \mathrm{mL}$, $\mu=42.8 \mathrm{cP})$.

On top of the sucrose solution, $500-1000 \mu \mathrm{L}$ of the optimized MLNC suspension was layered, followed by centrifugation at $25^{\circ} \mathrm{C}$ and $2000 \times g$ for $1 \mathrm{~h}$. The resultant fractions were carefully collected with a dispenser. The high concentration of sucrose makes the collected fractions unsuitable for examination using TEM and accordingly for assessing their quality. To remove the excess sucrose, an equal volume of $1 \mathrm{mM}$ phosphate buffer was added to the samples of selected fractions of MLNCs; the resulting suspensions were centrifuged for $15 \mathrm{~min}$ at $2000 \times \mathrm{g}$. The supernatant was discarded, the pellet was resuspended in $1 \mathrm{mM}$ phosphate buffer, and this sample was analyzed via TEM and DLS.

The TEM analysis of the fractions obtained via centrifugation in the $58 \%$ sucrose solution showed that the middle and upper fractions were very similar because target MLNCs constituted the bulk of each sample (Figure 8A,B). By contrast, the bottom fraction mainly consisted of large aggregates of the core nanoparticles enclosed in a thin lipid envelope and aggregates of naked core nanoparticles (Figure 8C). 


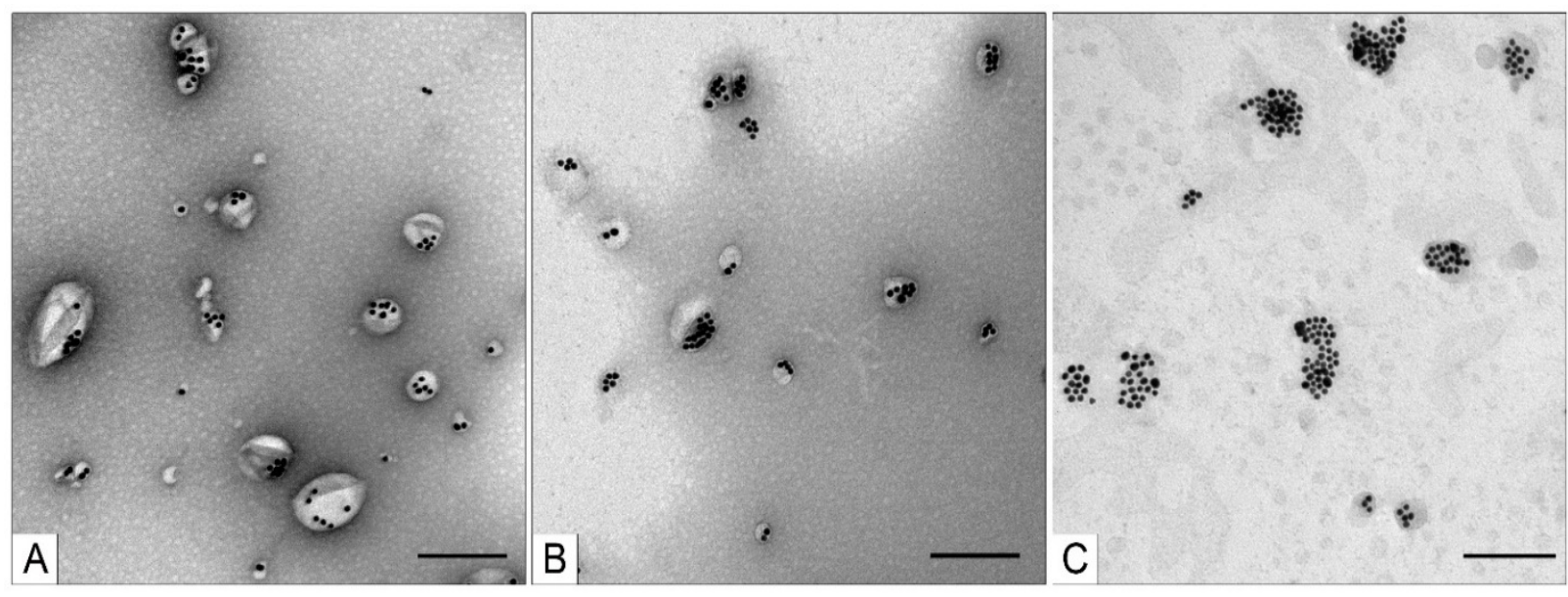

Figure 8. Ultrastructure of the fractions of optimized MLNCs obtained via centrifugation in a $58 \%$ sucrose solution for $1 \mathrm{~h}$ at $25{ }^{\circ} \mathrm{C}$ and $2000 \times g$. (A) Upper fraction, (B) middle fraction, and (C) bottom fraction. The scale bars correspond to $200 \mathrm{~nm}$. Negative staining with a $0.5 \%$ uranyl acetate solution followed by TEM.

We evaluated the yield of target MLNCs after all the steps of fabrication and purification. For this purpose, the gold content of the target fraction was calculated from its optical density and then divided by the amount of gold in the AuNPs used for the synthesis of the core nanoparticles. The yield of MLNCs (in terms of gold) in the middle fraction (Figure $8 \mathrm{~B}$ ) was $15 \%$; the sample after the removal of excess sucrose contained $5.5 \%$ of sucrose, and the hydrodynamic diameter of particles in this fraction was $195 \pm 78 \mathrm{~nm}$ with a PDI of 0.173 .

The low yield of MLNCs may be related both to the low efficiency of coating of the core nanoparticles during the fabrication of MLNCs and to degradation of the MLNC sample during the fractionation because the heavy gold core can "pierce" the lipid envelope during the centrifugation. Consequently, centrifugation duration was reduced to $30 \mathrm{~min}$, which yielded four fractions represented by colored discrete rings. Characteristics of these fractions are given in Table 3. TEM analysis of the fractions did not reveal any appreciable differences in the composition and structure of their nanoparticles as compared with the MLNC preparations after $1 \mathrm{~h}$ centrifugation.

Table 3. Physicochemical characteristics of optimized MLNCs after fractionation at $2000 \times g$ for $30 \mathrm{~min}$.

\begin{tabular}{cccc}
\hline Sample & Hydrodynamic Diameter, $\mathbf{n m}$ & PDI & Gold Content, $\%$ \\
\hline Optimized MLNCs & $152 \pm 75$ & $0.205 \pm 0.008$ & - \\
Upper fraction & $215 \pm 108$ & $0.198 \pm 0.011$ & 12 \\
Middle fraction & $397 \pm 163$ & $0.160 \pm 0.005$ & 32 \\
Bottom fraction & $1259 \pm 461$ & $0.097 \pm 0.009$ & 24 \\
Total gold content & & & 68 \\
\hline
\end{tabular}

The total gold content of the four fractions was $68 \%$ of the initial gold amount in AuNPs (utilized for the synthesis of core nanoparticles). One-third of the gold (apparently in the form of naked core nanoparticles released during the disintegration of MLNCs) was distributed throughout the rest $(10 \mathrm{~mL})$ of the sucrose solution volume in the centrifuge tube and was not detectable by direct observation.

In search of optimal fractionation duration, we reduced the centrifugation duration to $15 \mathrm{~min}$ and noticed that this time enables MLNCs' separation into two discrete fractions. The upper fraction contained 13-15\% of MLNCs, which corresponds to the upper fraction with the half-hour centrifugation. At the same time, an increase in the concentration of 
MLNCs up to $40 \%$ was observed in the middle fraction. It is this fraction (Figure 9) that we are currently testing in cell culture assays.

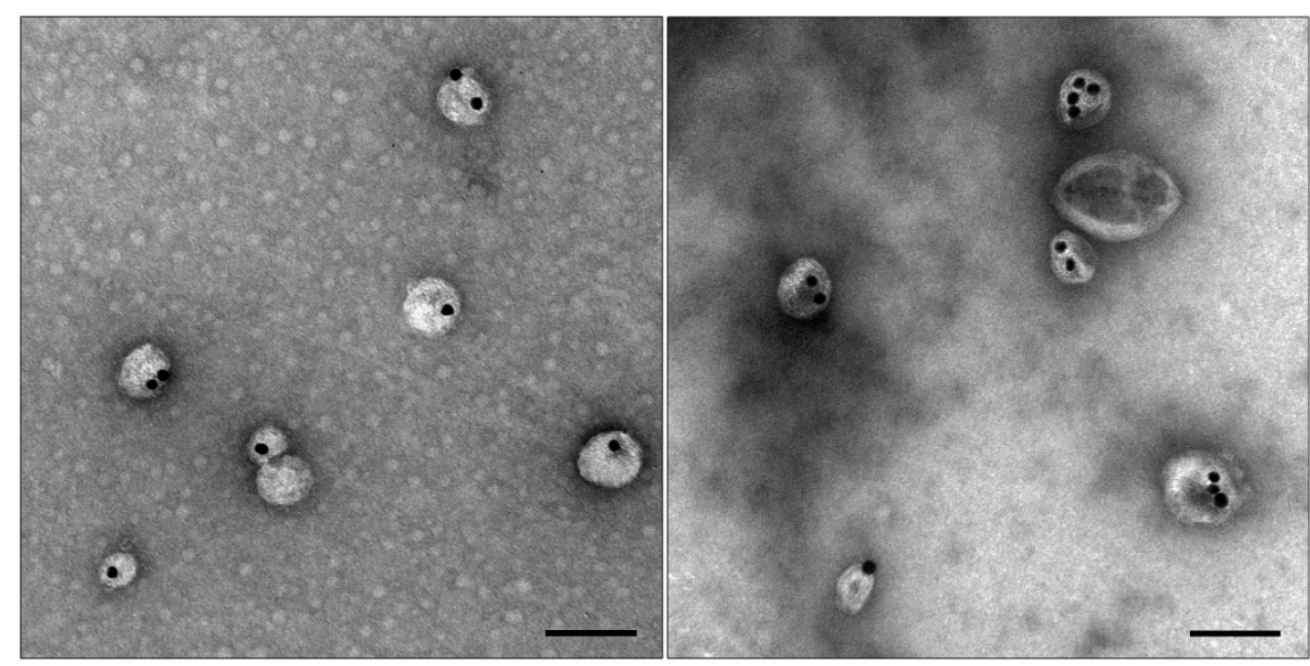

Figure 9. Ultrastructure of optimized MLNCs after $15 \mathrm{~min}$ fractionation in 58\% sucrose and removal of excess sucrose. The scale bars correspond to $100 \mathrm{~nm}$. Negative staining with a $0.5 \%$ uranyl acetate solution followed by TEM.

Our study indicates that the amount of target MLNCs in the final suspension is determined not only by the efficiency of coating of the core nanoparticles with the lipid envelope but also by MLNC preservation during the purification and concentration procedures. We believe that the approaches that we used to improve the quality of lipid-coated nanoconstructs and the newly developed methodology will be useful to researchers creating similar nanoconstructs.

Undoubtedly, an important factor in the proposed method is the choice of a medium for separating a suspension of MLNCs into fractions. In addition to sucrose and cesium chloride, we tested another type of viscous medium: aqueous solutions of glycerin (details are given in the Materials and Methods section).

The best results were obtained with the following parameters of the procedure: centrifugation in a $75 \%$ glycerol solution for $40 \mathrm{~min}$ at $8000 \times g$ and $25^{\circ} \mathrm{C}$ with subsequent dialysis for $16 \mathrm{~h}$ (using membranes for extruders, pore diameter $30 \mathrm{~nm}$ ). As in the case of sucrose, in glycerol solutions, optimized MLNCs get separated into fractions (Figure 10A). According to TEM (Figure 10B), the upper fraction contained lipid particles and vesicles (hydrodynamic diameter $123 \pm 55 \mathrm{~nm}$, PDI =0.366), the middle dark-red fraction contained MLNCs (hydrodynamic diameter $127 \pm 40 \mathrm{~nm}, \mathrm{PDI}=0.279$ ), and the bottom fraction consisted of large aggregates of the core nanoparticles enclosed in an "incomplete" lipid envelope and aggregates of core nanoparticles stuck to fragments of lipid envelopes (hydrodynamic diameter $233 \pm 74 \mathrm{~nm}$, PDI $=0.280$ ).

We fine-tuned all stages of the fractionation of optimized MLNCs in glycerol solutions, subsequent purification via dialysis, and the concentration procedure (data not shown). The highest-quality end products were homogeneous, had a PDI of $\sim 0.160$, and contained $\geq 22-27 \%$ of glycerol as well as nanoparticles with a hydrodynamic diameter of $115 \pm 49 \mathrm{~nm}$. There were concerns that the presence of glycerol could negatively affect cell viability, and we performed an assay of its cytotoxicity on cultured SC-1 R780 fibroblasts.

It turned out that when a $75 \%$ solution of glycerol is diluted 32 -fold (down to $2.25 \%$ concentration) - Which corresponds to its calculated concentration when the final MLNC suspension is added to the cell culture-A negative effect on cell viability is detectable. By contrast, sucrose solutions did not manifest pronounced toxicity toward the cultured fibroblasts at the corresponding dilutions (Figure 11). 


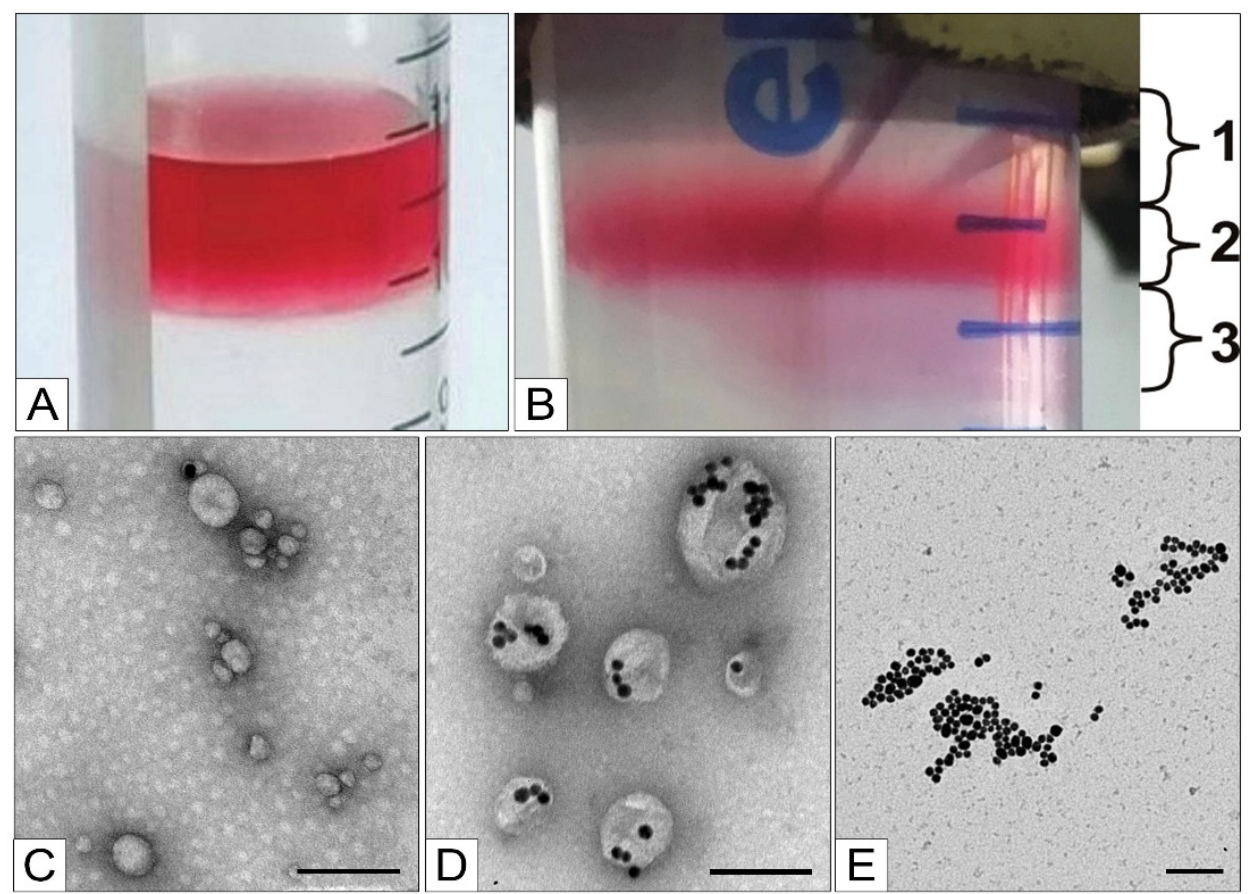

Figure 10. Top row: purification of optimized MLNCs via centrifugation in $75 \%$ glycerin. (A) The sample is applied to a glycerin layer; (B) separation of the sample into three fractions after centrifugation (1: upper fraction, 2: middle (target) fraction, and 3: bottom fraction). Bottom row: fractions of optimized MLNCs obtained via centrifugation in $75 \%$ glycerol and purified via dialysis: (C) upper fraction, (D) middle fraction, and (E) bottom fraction. The scale bars correspond to $100 \mathrm{~nm}$. Contrasting by means of a $0.5 \%$ uranyl acetate solution followed by TEM.

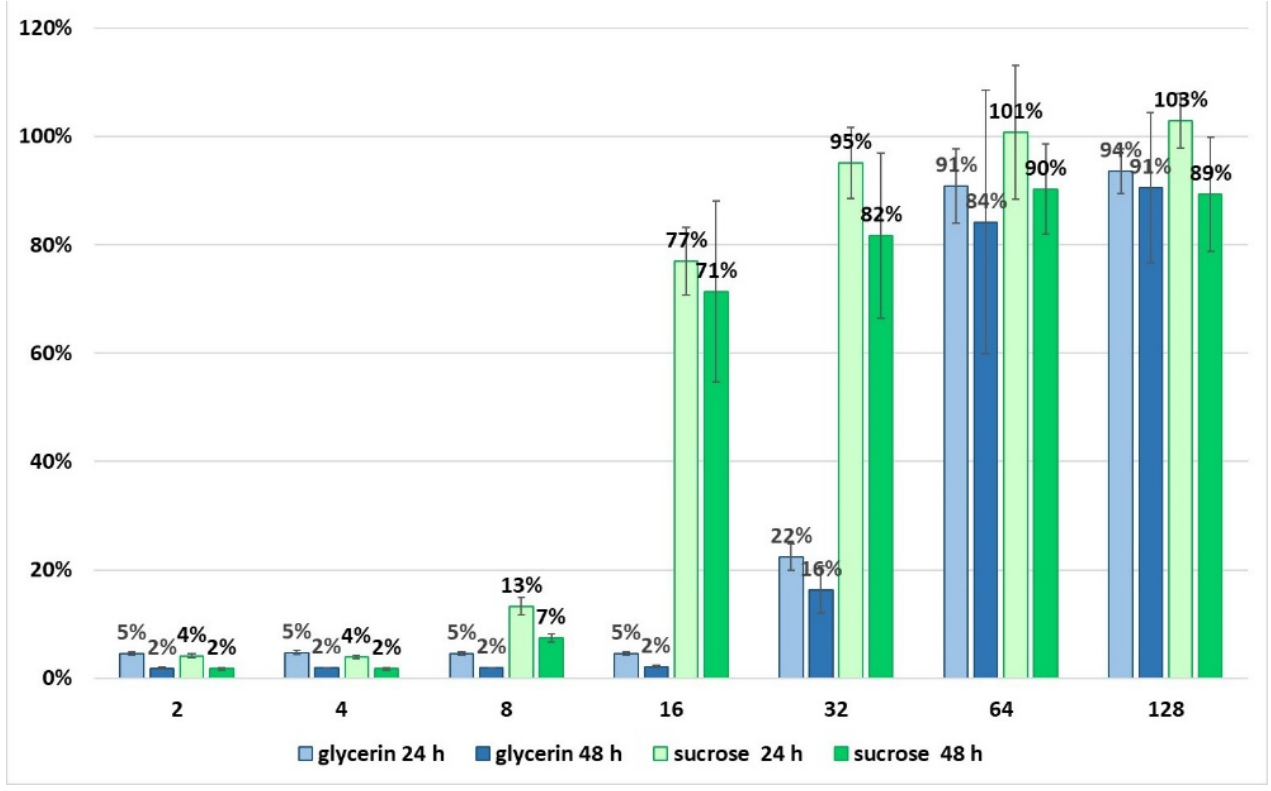

Figure 11. Viability of SC-1 R780 fibroblasts in the presence of glycerol or sucrose. The vertical axis denotes the percentage of viable cells, and the horizontal axis shows fold dilution of stock solutions of glycerol $(75 \%)$ and sucrose $(58 \%)$.

These findings indicate that the presence of glycerol in the culture medium, even at low concentrations, has an adverse effect on cells. Therefore, the MLNC preparations obtained using the purification method involving fractionation of samples in glycerol is 
not suitable for research on cultured cells. We present the results of this study to draw the readers' attention to the necessity of comprehensive characterization before the approval of methods intended for nanobiotechnology and nanomedicine.

\section{Conclusions}

Lipid-coated particles that serve as a carrier of siRNA are the subject of numerous studies. Several years ago, we published the proof of principle for the construction of an AuNP-based MLNC that efficiently delivers siRNA into the cell [15]. Nonetheless, we were not satisfied with the quality of the obtained nanocomposites, and thus, here we found some ways to improve it in comparison with the original version. In this work, we demonstrated that even seemingly insignificant modifications of the steps of the MLNC fabrication affect end product quality. For example, the optimal reaction mixture for obtaining the core nanoparticles contains $0.1 \mathrm{mM} \mathrm{MgSO}_{4}$ and $5 \mathrm{mM} \mathrm{NaCl}$; lipid film synthesis at $12 \mathrm{mmHg}$ without thermostatting improves the quality of the forming MLNCs, as does the assembly of MLNCs in $1 \mathrm{mM}$ phosphate buffer. Having optimized all the steps of MLNC fabrication, we noticed that $15 \mathrm{~min}$ centrifugation at $2000 \times \mathrm{g}$ in $58 \%$ sucrose yields a fraction containing $40 \%$ of target MLNCs, i.e., a doubled proportion of these nanoconstructs as compared to the end product of the original procedure [15].

We think that this study can help researchers who design nanoconstructs based on metal nanoparticles coated with a lipid envelope.

Author Contributions: Conceptualization, I.A.P., I.S.D. and A.V.E.; methodology, I.S.D.; investigation, A.V.E., J.E.P., I.S.D. and B.P.C.; resources, D.V.P.; data curation, I.A.P.; writing-Original draft preparation, A.V.E., I.S.D. and I.A.P.; writing-Review and editing, E.I.R.; visualization, J.E.P.; supervision, D.V.P.; project administration, E.I.R.; funding acquisition, E.I.R. All authors have read and agreed to the published version of the manuscript.

Funding: This research was funded by the Russian Science Foundation, grant \# 19-15-00217; the synthesis of siRNA was funded by Russian State Funded Project \# 121031300042-1.

Data Availability Statement: Data are available on request from the corresponding author.

Conflicts of Interest: The authors declare no conflict of interest.

\section{References}

1. Kulkarni, J.A.; Witzigmann, D.; Thomson, S.B.; Chen, S.; Leavitt, B.R.; Cullis, P.R.; van der Meel, R. The current landscape of nucleic acid therapeutics. Nat. Nanotechnol. 2021, 630, 630-643. [CrossRef] [PubMed]

2. Margus, H.; Arukuusk, P.; Langel, Ü.; Pooga, M. Characteristics of Cell-Penetrating Peptide/Nucleic Acid Nanoparticles. Mol. Pharm. 2015, 13, 172-179. [CrossRef]

3. Elsabahy, M.; Nazarali, A.; Foldvari, M. Non-Viral Nucleic Acid Delivery: Key Challenges and Future Directions. Curr. Drug Deliv. 2011, 8, 235-244. [CrossRef] [PubMed]

4. Zhou, S.; Chen, W.; Cole, J.; Zhu, G. Delivery of nucleic acid therapeutics for cancer immunotherapy. Med. Drug Dicovery 2020, 6, 100023. [CrossRef] [PubMed]

5. Gupta, A.; Andresen, A.J.; Manan, R.S.; Langer, R. Nucleic Acid Delivery for Therapeutic Applications. Adv. Drug Deliv. Rev. 2021. Ahead of print. [CrossRef] [PubMed]

6. Berger, M.; Lechanteur, A.; Evrard, B.; Piel, G. Innovative lipoplexes formulations with enhanced siRNA efficacy for cancer treatment: Where are we now? Int. J. Pharm. 2021, 605, 120851. [CrossRef] [PubMed]

7. Rinoldi, C.; Zargarian, S.S.; Nakielski, P.; Li, X.; Liguori, A.; Petronella, F.; Presutti, D.; Wang, Q.; Costantini, M.; De Sio, L.; et al. Nanotechnology-Assisted RNA Delivery: From Nucleic Acid Therapeutics to COVID-19 Vaccines. Small Methods 2021, 5, 2100402. [CrossRef]

8. Aghamiri, S.H.; Raee, P.; Talaei, S.; Mohammadi-Yeganeh, S.; Bayat, S.H.; Rezaee, D.; Ghavidel, A.A.; Teymouri, A.; Roshanzamiri, S.; Farhadi, S.H.; et al. Nonviral siRNA delivery systems for pancreatic cancer therapy. Biotechnol. Bioeng. 2021, 118, 3669-3690. [CrossRef]

9. Shaabani, E.; Sharifiaghdam, M.; De Keersmaecker, H.; De Rycke, R.; De Smedt, S.; Faridi-Majidi, R.; Braeckmans, K.; Fraire, J.C. Layer by Layer Assembled Cihitosan-Coated Gold Nanoparticles for Enhanced siRNA Delivery and Silencing. Int. J. Mol. Sci. 2021, 22, 831. [CrossRef]

10. Sharifiaghdam, M.; Shaabani, E.; Sharifiaghdam, Z.; De Keersmaecker, H.; De Rycke, R.; De Smedt, S.; Faridi-Majidi, R.; Braeckmans, K.; Fraire, J.C. Enhanced siRNA Delivery and Selective Apoptosis Induction in H1299 Cancer Cells by Layer-byLayer-Assembled Se Nanocomplexes: Toward More Efficient Cancer Therapy. Front. Mol. Biosci. 2021, 8, 639184. [CrossRef] 
11. Bonoiu, A.C.; Mahajan, S.D.; Ding, H.; Roy, I.; Yong, K.-T.; Kumar, R.; Hu, R.; Bergey, E.J.; Schwartz, S.A.; Prasad, P.N. Nanotechnology approach for drug addiction therapy: Gene silencing using delivery of gold nanorodsiRNA nanoplex in dopaminergic neurons. Proc. Natl. Acad. Sci. USA 2009, 106, 5546-5550. [CrossRef]

12. Bai, X.; Wang, Y.; Song, Z.; Feng, Y.; Chen, Y.; Zhang, D.; Feng, L. The Basic Properties of Gold Nanoparticles and their Applications in Tumor Diagnosis and Treatment. Int. J. Mol. Sci. 2020, 21, 2480. [CrossRef]

13. Li, W.; Cao, Z.H.; Liu, R.; Liu, L.; Lia, H.; Li, X.; Chen, Y.; Lu, C.H.; Liu, Y. AuNPs as an important inorganic nanoparticle applied in drug carrier systems. Artif. Cells Nanomed. Biotechnol. 2019, 47, 4222-4233. [CrossRef]

14. Lopes, T.S.; Alves, G.G.; Pereira, M.R.; Granjeiro, J.M.; Leite, P.E.C. Advances and potential application of gold nanoparticles in Nanomedicine. J. Cell Biochem. 2019, 120, 16370-16378. [CrossRef]

15. Poletaeva, J.; Dovydenko, I.; Epanchintseva, A.; Korchagina, K.; Pyshnyi, D.; Apartsin, E.; Ryabchikova, E.; Pyshnaya, I. NonCovalent Associates of siRNAs and AuNPs Enveloped with Lipid Layer and Doped with Amphiphilic Peptide for Efficient siRNA Delivery. Int. J. Mol. Sci. 2018, 19, 2096. [CrossRef]

16. Tschuch, C.; Schulz, A.; Pscherer, A.; Werft, W.; Benner, A.; Hotz-Wagenblatt, A.; Barrionuevo, L.S.; Lichter, P.; Mertens, D. Off-target effects of siRNA specific for GFP. BMC Mol. Biol. 2008, 9, 60. [CrossRef] [PubMed]

17. Pavlova, A.S.; Yakovleva, K.I.; Epanchitseva, A.V.; Kupryushkin, M.S.; Pyshnaya, I.A.; Pyshnyi, D.V.; Ryabchikova, E.I.; Dovydenko, I.S. An Influence of Modification with Phosphoryl Guanidine Combined with a 20-O-Methyl or 20-Fluoro Group on the Small-Interfering-RNA Effect. Int. J. Mol. Sci. 2021, 22, 9784. [CrossRef] [PubMed]

18. Shashkova, V.V.; Epanchintseva, A.V.; Vorobjev, P.E.; Razum, K.V.; Ryabchikova, E.I.; Pyshnyi, D.V.; Pyshnaya, I.A. Multilayer Associates Based on Oligonucleotides and Gold Nanoparticles. Rus. J. Bioorg. Chem. 2017, 43, 64-70. [CrossRef]

19. Epanchintseva, A.; Vorobjev, P.; Pyshnyi, D.; Pyshnaya, I. Fast and Strong Adsorption of Native Oligonucleotides on CitrateCoated Gold Nanoparticles. Langmuir 2018, 34, 164-172. [CrossRef] [PubMed]

20. Liu, X.; Atwater, M.; Wang, J.; Huo, Q. Extinction coefficient of gold nanoparticles with different sizes and different capping ligands. Colloids Surf. B 2007, 58, 3-7. [CrossRef] [PubMed]

21. Rodr1'guez-Corrales, J.A.; Josan, J.A. Resazurin Live Cell Assay: Setup and Fine-Tuning for Reliable Cytotoxicity Results. Methods Mol. Biol. 2017, 1647, 207-219.

22. Epanchintseva, A.V.; Poletaeva, J.E.; Pyshnyi, D.V.; Ryabchikova, E.I.; Pyshnaya, I.A. Long-term stability and scale-up of noncovalently bound gold nanoparticle-siRNA suspensions. Beilstein J. Nanotechnol. 2019, 10, 2568-2578. [CrossRef] [PubMed]

23. Epanchintseva, A.; Dolodoev, A.; Grigoreva, A.; Chelobanov, B.; Pyshnyi, D.; Ryabchikova, E.; Pyshnaya, I. Non-covalent binding of nucleic acids with gold nanoparticles provides their stability and effective desorption in environment mimicking biological media. Nanotechnology 2018, 29, 355601. [CrossRef] [PubMed]

24. Menhaj, A.B.; Smith, B.D.; Liu, J. Exploring the thermal stability of DNA-linked gold nanoparticles in ionic liquids and molecular solvents. Chem. Sci. 2012, 3, 3216. [CrossRef]

25. Zhang, Z.; Li, H.; Zhang, F.; Wu, Y.; Guo, Z.; Zhou, L.; Li, J. Investigation of halide-induced aggregation of Au nanoparticles into spongelike gold. Langmuir 2014, 30, 2648-2659. [CrossRef] [PubMed]

26. Liu, B.; Kelly, E.Y.; Liu, J. Cation-size-dependent DNA adsorption kinetics and packing density on gold nanoparticles: An opposite trend. Langmuir 2014, 30, 13228-13234. [CrossRef]

27. Federal Medical \& Biological Agency. Research Institute of Physical-Chemical Medicine. Website of Extracellular Vesicles Research Group. Centrifugation Calculator. Available online: http:/ / vesicles.niifhm.ru (accessed on 30 September 2021). 